\title{
Progress of the ECRH Upper Launcher design for ITER*
}

\author{
D. Strauss ${ }^{\mathrm{a},{ }^{* *}}$, G. Aiello ${ }^{\mathrm{a}}$, A. Bruschi ${ }^{\mathrm{c}}$, R. Chavan ${ }^{\mathrm{b}}$, D. Farina ${ }^{\mathrm{c}}$, L. Figini ${ }^{\mathrm{c}}$, M. Gagliardi ${ }^{\mathrm{h}}$, V. Garcia ${ }^{\mathrm{h}}, \mathrm{T}$. \\ P. Goodman ${ }^{b}$, G. Grossetti ${ }^{\mathrm{a}}$, C. Heemskerk ${ }^{\mathrm{g}}$, M. A. Henderson ${ }^{\mathrm{i}}, \mathrm{W}$. Kasparek ${ }^{\mathrm{t}}$, A. Krause ${ }^{\mathrm{b}}$, J.-D.

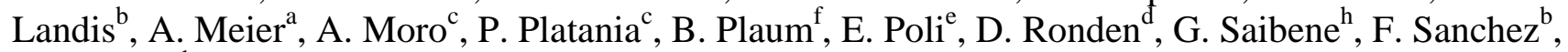 \\ O. Sauter ${ }^{b}$, T. Scherer ${ }^{a}$, S. Schreck ${ }^{a}$, A. Serikov ${ }^{a}$, C. Sozzi ${ }^{c}$, P. Spaeh ${ }^{a}$, A. Vaccaro ${ }^{a}$, B. Weinhorst ${ }^{a}$
}

\author{
${ }^{a}$ Karlsruhe Institute of Technology, Assoc. KIT-EURATOM, D-76021 Karlsruhe, Germany \\ ${ }^{b}$ Centre de Recherches en Physique des Plasmas, CRPP-EPFL, CH-1015 Lausanne, Switzerland \\ ${ }^{c}$ Istituto di Fisica del Plasma CNR, Euratom Association, 20125 Milano, Italy \\ ${ }^{d}$ FOM Institute DIFFER, P.O. Box 1207, 3430 BE Nieuwegein, Netherlands \\ ${ }^{e}$ Max-Planck-IPP, Euratom Association, D-85748 Garching, Germany \\ ${ }^{f}$ Institut für Plasmaforschung, IPF, D-70569 Stuttgart, Germany \\ ${ }^{g}$ Heemskerk Innovative Technology, Merelhof 2, 2172 HZ, Sassenheim, Netherlands \\ ${ }^{h}$ Fusion for Energy, Barcelona \\ ${ }^{i}$ ITER Organization, 13108 Saint-Paul-lez-Durance, France
}

\begin{abstract}
The design of the ITER ECRH system provides 20MW millimeter wave power for central plasma heating and MHD stabilization. The system consists of an array of 24 gyrotrons with power supplies coupled to a set of transmission lines guiding the beams to the four upper and the equatorial launcher. The front steering upper launcher design described herein has passed successfully the preliminary design review, and it is presently in the final design stage. The launcher consists of a millimeter wave system and steering mechanism with neutron shielding integrated into an upper port plug with the plasma facing blanket shield module (in-vessel) and a set of ex-vessel waveguides connecting the launcher to the transmission lines.
\end{abstract}

Part of the transmission lines are the ultra-low loss CVD torus diamond windows and a shutter valve, a mitre bend section and the feedthroughs integrated in the plug closure plate. These components are connected by corrugated waveguides and form together the first confinement system (FCS). In-vessel, the mm-wave system includes a quasi-optical beam propagation system including four mirror sets and a front steering mirror. The millimeter wave system is integrated into a specifically optimized upper port plug providing structural stability to withstand plasma disruptions forces and the high heat load from the plasma side with a dedicated blanket shield module. A recent update in the ITER interface definition has resulted in the recession of the upper port plug first wall panels, which is now integrated into the design. Apart from the millimeter wave system the upper port plug houses also a set of shield blocks which provide neutron shielding. An overview of the actual ITER ECRH upper launcher is given together with some highlights of the design.

Keywords: ITER, ECRH, Launcher, Blanket, Diamond Windows, Front Steering

\section{Introduction}

The four ITER ECRH Upper Launchers (UL, see figure 1 and [1]) are plasma facing port plugs located in the ITER upper ports, their development is driven by ITER with European support by F4E. The Upper Launcher design is developed by the European ECH Upper Launcher Consortium of Associations ECHULCA (KIT/Germany, CNR/Italy, CRPP/Switzerland, Differ/Netherlands, IPP and IPF/Germany). The upper launchers are connected via transmission lines [2] to a set of gyrotrons providing up to $20 \mathrm{MW}$ millimeter wave power at a fixed frequency of $170 \mathrm{GHz}$ [3].

The main purpose of the upper launchers is to drive local current with the aim to suppress neoclassical tearing modes which on one side can trigger plasma disruptions generating loads on the components close to the plasma and on the other side lead to confinement degradation. The coupling of the fixed frequency millimeter waves to the plasma via electron cyclotron requires beam steering realized by front steering mechanisms to match resonance and local heating (electron cyclotron resonant heating, ECRH). The upper launcher millimeter wave power injection system is complemented by an equatorial launcher (JAEA, [4]) for central plasma heating and off-axis current drive. This publication gives an overview of the developments towards final design since the preliminary design review of the ITER ERCH Upper Launchers [1]. The technical requirements describe criteria which should be fulfilled to obtain a working design. Their fulfillment however is not yet a guarantee to reach the ideal of a good design. The selected design updates show improvements of the design towards this ideal based on simple reliability,

* This work was supported by Fusion for Energy under the grant contract No. F4E-2010-GRT-161 and by ITA C52TD39FE. The views and opinions expressed herein reflect only the author's views. Fusion for Energy and ITER are not liable for any use that may be made of the information contained therein.

** corresponding author: dirk.strauss@kit.edu 
availability, maintainability and inspectability (RAMI) considerations. The design validation finally provides criteria to prove the design by means of numerical simulations, prototyping and testing.

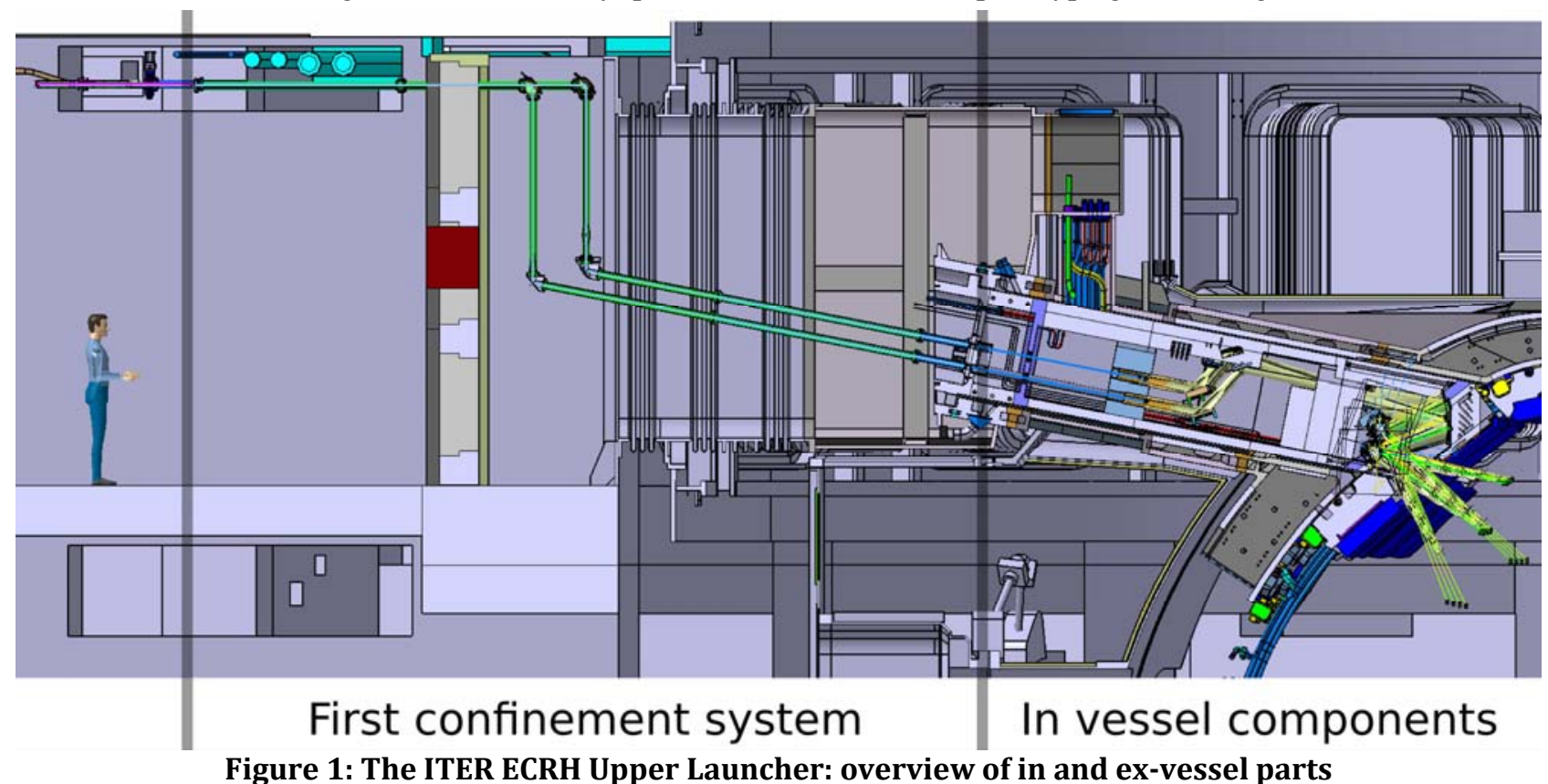

\section{Requirements}

Two requirement categories for the UL design can be defined: on one side the operational ones shall guarantee the component integrity withstanding operational loads fulfilling reliability and maintainability criteria. On the other side functional requirements must be fulfilled which are in the case of the ITER ECRH upper launchers MHD instability stabilization of the neoclassical tearing modes (NTMs) avoiding confinement degradation. Compared to other components the two requirement categories are coupled for the upper launchers: if the NTM can't be stabilized, which is the main functional purpose of the ULs, a disruption might be triggered with severe loads an all in-vessel components; quality and quantity of disruptions might affect their operational integrity. It can be concluded that the functional requirements on the ULs are entangled with operational requirement not only for the UL itself, but also for the other in-vessel components. In the following a brief description of the main functional requirements and an overview of the operational ones are given.

The NTM evolution is usually calculated using the generalized Rutherford equation. Two criteria [5], derived from this equation, have been proposed to describe the requirements for local current drive and hence the UL performance. First the locally driven current density should exceed the unperturbed bootstrap current density by a factor of 1.2 at the flux surface where the mode develops. Second the driven current density multiplied by the full width of the deposition profile and divided by the bootstrap current density should be larger than $5 \mathrm{~cm}$. These two criteria impose geometrical requirements on the optical system of the UL as focal points size and depth of focus as well as poloidal and toroidal injection angles. They directly affect the design of the plasma facing components, the
ECRH port plug blanket shield module with its first wall panel as well as neighboring blankets which need to have specific cut-outs to allow a line of sight access of the beam to the location of the instability in the plasma. Cut outs in the ITER first wall have to be designed preserving the shielding function of the blankets. Only a matching intersection between functional and operational requirements can fulfill the ideal of the UL design and it is very challenging to find such a working intersection. It can be concluded that here physics and tokamak design constraints give the lowest degree of design freedom and the design of the entire upper launchers is based on the fulfillment of these criteria defining also key requirements for transmission lines and gyrotrons. The optical and structural design close to the first wall can therefore be identified as the major challenge on the ITER ECRH Upper Launcher design.

First wall cut outs and the plasma injection angles determine the design of the optical system in order to access the location of the $q=2 / 1$ and 3/2 rational surfaces. The beam paths are constrained by the interfaces to the neighbouring components in the port and the corresponding wall thickness needed for the structural system. Specific design requirements arise e.g. for the velocity of the steering mirror mechanism to access the instability in time or for ultra low loss CVD diamond windows to assure tritium confinement for which applicable nuclear codes and standards are not available. Additionally, active cooling of structural and optical systems has to be foreseen as well as sufficient neutron shielding and the component maintainability. The design has further to fulfill load requirements during normal operation and during off-normal events such as plasma disruptions, earth quakes etc. A large number of these events, defined by the ITER load specifications and the EC-UL specific loads, have to be considered as well as combined loads during the design validation process. 
High requirements have to be fulfilled as ITER is a nuclear facility including the demonstration the tritium confinement of the ex-vessel components, and this is a further huge challenge for the launcher design.

The interfaces to neighbouring components determine the geometrical constraints of the launcher design including the cut outs at neighbouring blankets. An update of these beam paths might require an interface change for the affected blankets. A clear definition of these interfaces and their validity ideally over the time of the final design development is a key requirement in the hands of the ITER team and the design teams for an effective design progress allowing a proper definition of the constraints, based on which the designers develop a matching intersection between operational and functional requirements.

\section{Design status}

The design development is based on the preliminary design of the four upper launchers [1] and the actual intermediate design is shown in figure 2.

Eight transmission lines transport the beam into each launcher with diamond windows as the first tritium barrier, after a transition to a quasi-optical system two front steering mirror units give access to the resonance location on the targeted magnetic flux surface.

The final procedure for a generic maintenance solution, namely the removal of ECRH and diagnostics upper port plugs with the help of a port plug cask system is still under work and therefore interface changes, e.g. by the introduction of rails in the plug-port interspace with a potential impact on both the optical and structural design, are probable. This uncertainty led to the decision to continue the design with an emphasis on the first confinement system (FCS), which has its criticality due to tritium confinement and nuclear codes and standards fulfillment, accompanied by a design update of the invessel part. As a consequence the upper launcher final design review was split into two parts: first the final design of the FCS is planned, and after a second design iteration of the in-vessel components the remaining second final design review is foreseen. This means a compromise between the urge of finalizing the first wall design, not yet fixed interfaces to the upper launcher and resolving the high requirements on tritium confinement.

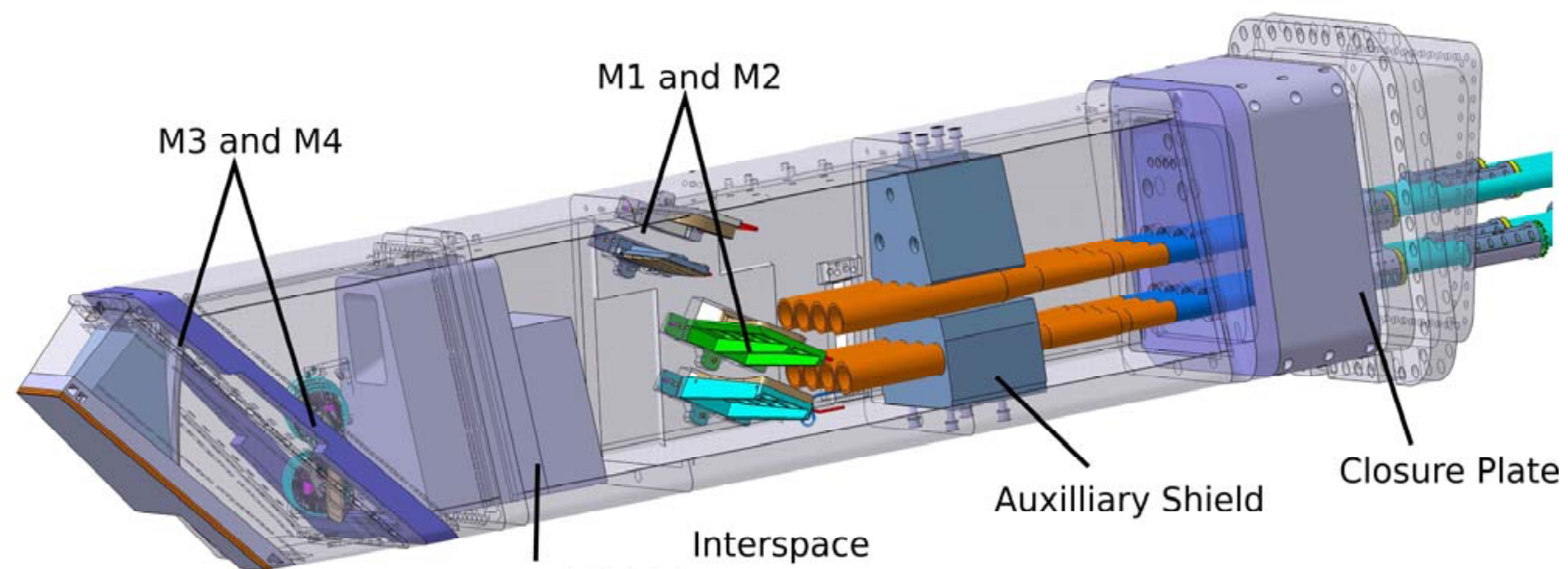

\section{Blanket Shield Module Internal Shield with First Wall Panel}

Figure 2: In-vessel part of the ECRH Upper Launcher

\subsection{First confinement system}

The first confinement system of the UL starts with the CVD torus diamond windows [6] forming the interface to the transmission line section developed by the US Domestic Agency. The waveguide section includes shutter valves protecting the transmission lines in the case of a window failure. Mitre bends form a dogleg beam path followed by a waveguide section ending at the UL closure plate. The connection to the closure plate is formed by a double helicoflex sealed sub-plate. It allows the removal of the entire set of eight waveguides and their connected counter pieces including tapers placed inside the launcher.

The actual design has been validated for different aspects including seismic loads [7] and thermal expansion from room temperature to operating temperature and vacuum vessel bake out conditions. The analysis showed that the waveguide couplings initially foreseen are too stiff to compensate for the thermal expansion. With the aim to avoid bellows in the transmission lined it was decided to develop more flexible waveguide components in the planned next design iteration. As a further design progress, the formerly individual mitre bends placed in each waveguide section are to be replaced by mitre bend blocks. They have the further advantage of stabilizing the ex-vessel ensemble against seismic events.

CVD diamond windows [8] are a key component of the ITER ECRH system. In today's knowledge only diamond provides the required material properties as transparency, mechanical strength and thermal conductivity for the use in the high power millimeterwave system. For the four upper launchers and the 
equatorial launcher a set of 56 CVD torus diamond windows for tritium confinement are used. Additional 24 diamond windows are foreseen for the protection of the gyrotron vacuum. Each diamond disk is optimized for the fixed frequency of $170 \mathrm{GHz}$, the torus window disks have a thickness of $1.11 \mathrm{~mm}$, a beam aperture of $63.5 \mathrm{~mm}$ and a weight of 86 karat. The actual torus diamond window design is considered mature and the final prototyping and testing is being initiated. A new development has been started with the integration of a detector array, which can be integrated into the torus window. The aim is to detect coupling of higher order modes through the cylindrical side face of the diamond disk for beam profile reconstruction and to detect a potential crack in the diamond disk.

For the subplate waveguide feedthroughs several options as welded solutions with or without individual maintenance, helicoflex sealed waveguide feedthroughs have been checked. The favorite solution is a monoblock with geometrically staged helicoflex sealed connectors to the waveguides. The minimization of SIC-1 welds has been a major design driver for this development with the limited space as a major design constraint.

The closure plate was retracted close to the port plug seal compared to the PDR design [9]; with this simple modification a number of SIC-1 welds is now located in the in-vessel part no longer forming a first confinement barrier. Further the maintenance access to the closure plate feedthroughs is now significantly simplified.

\subsection{In vessel part of the upper launcher}

In parallel to the progress on the final design of the first confinement system also the in-vessel components are being developed. The UL has a trapezoidal shape with the blanket shield module (BSM) connected via a bolted flange. The UL is connected with a flange on its back end to the port with lip seals and a closure plate for tritium confinement. The closure plate has feedthroughs for cooling lines, the waveguides and the Helium supply lines driving the front steering mirror mechansim. An actively cooled auxiliary shield in the rear part serves for neutron shielding and fixes the waveguide tapers at their transition to the quasi-optical propagation. To minimize neutron streaming, two mirror sets form a dogleg beam path through the internal shield, which is located in the front section of the trapezoidal main frame. The side wall of the main frame serves as an optical bench, on which the mirror sets M1, M2 and M3 are fixed. The focusing mirror unit M3 is integrated into the BSM.

Availability considerations lead to the need for efficient maintenance solutions, usually by remote handling [10]. Two mainly independent approaches can be defined; on one side the general reliability of tools and processes can be optimized, on the other side the reduction of amount and complexity of the required remote handling tasks leads to faster and more reliable maintenance operations. If a subcomponent is deeply nested in a subcomponent tree requiring a serial remote handling approach, then the ideal is to break up the nested subcomponent tree by design to independently maintained subcomponents. These can be ideally accessed directly converting the serial $\mathrm{RH}$ operations approach to parallel ones, strongly reducing the complexity of the maintenance process.

In the preliminary upper launcher design the maintenance of the mirror sets M1 and M2 required the removal of a bottom hatch in the plug main frame, a cutting of a number of water and He pipes for the BSM, the front mirrors and the internal shield directly above the hatch for obtaining access to the mirrors in a blocked geometrical configuration requiring several remote handling steps. This serial process could be broken up by design; the new solution [11] is to insert and remove the mirror sets fixed with captive bolts in small slits within the main frame wall directly from the outside including access for cutting and re-welding of only the attached cooling lines, resembling to minimally invasive surgery.

Another approach to reduce complexity and risks of maintenance operations is to carefully assess the need for remote handling for specific components. It is actually under investigation if it is sensible to suggest a downgrade of the auxiliary shield block in its remote handling class to a fixed installation for lifetime. The benefits would not only be a significant reduction of $\mathrm{RH}$ operations, also the bottom hatch for maintenance access might be revised or even become obsolete reducing design complexity and manufacturing costs.

The need for maintenance arises either from planned service intervals with an exchange of critical components reaching their lifetime or when a component fault or failure is assumed. The ideal component inspectability provides fault detection during operation before a failure occurs; the ideal robust design has sufficient fault to failure intervals allowing an early mitigation before a failure affects the system integrity. With the given boundary conditions such as radiative environment and very limited space for inspection tools it is very difficult and challenging, often out of the actual technical reach, to develop designs and inspection solutions close to this ideal. One compromise is to refrain from an on-line inspection and to use off-line inspection tools instead, during planned downtimes.

A specific failure mode for ECRH launchers is arcing caused by the millimeter wave beams, which can lead to high local thermal loads and melting of component parts. In the interspace between the auxiliary and the internal shield blocks, where the mirror sets M1 and M2 are located, the beams are no longer guided by waveguides. They enter via tapers to a quasi-optical (vacuum) beam propagation. Due to the local magnetic field strength in this interspace a resonance might trigger arcing. An early detection of such arcing as a fault condition would be highly beneficial; the time to failure interval could be used to mitigate the problem e.g. switching off the relevant beam lines relying on the other ULs. Two tubes running from a closure plate feedthrough to the M1/M2 interspace, passing through the auxiliary shield block, are being studied in the present design phase. The use of fibre optics is investigated with the ideal of having the on line inspection of arcing in the critical interspace. The 
system could be further explored as an off-line inspection tool if the fibre optics system is removed and instead a camera system is introduced for interspace and mirror set inspection. Without such a system only a complicated and very time consuming port plug removal with associated hot cell operations for the interspace fault inspection could provide similar information. This however is not foreseen for practical reasons such as availability and costs.

\section{Design validation}

Two categories of design validation are used for the ITER ECRH Upper Launcher design. The CATIA models are used for a set of numerical design analyses and its validation against codes \& standards. The simulations however have their limits in modeling assumptions such as the validity of material properties, especially after irradiation, or idealized joining techniques as well as load assumptions. Prototyping and testing are the complementing method for further validation covering manufacturing issues as costs, tolerances and weld qualities. Prototype test facilities have been set up; the Launcher Handling and Testing facility LHT at KIT provides water flows with fast temperature transients up to the bake out conditions with $240^{\circ} \mathrm{C}$ at $4.4 \mathrm{MPa}$ [15]. The facility has recently been extended with a large vacuum chamber able to house large launcher structural components. Another prototype testing facility has been built up at CRPP for the test of highly loaded millimeter wave components as the mirrors.

The quality of the numerical analyses has been significantly improved since the preliminary design review. Higher resolved MCNP analyses [12] and a direct interpolation of nodal results directly to the mesh of the FEM models give a better representation of volumetric neutron heat loads. In combination with CFD and thermal analyses the generated stresses are calculated now with higher accuracy [13]. The same approach has been successfully introduced for EM analyses [14]. Further the seismic analysis approach could be improved using response spectrum analysis with spatial combinations based on Newmark's rule [7]. Thermal expansion simulations of the ex-vessel system accompany the millimeterwave system design. The ITER load specifications define a huge set of possible load combinations; a subset of UL relevant load combinations for specific components is being developed and suggested to ITER.

\section{Conclusions}

Since the preliminary design review significant progress could be made for the upper launcher design and the validation tools. The experience and knowledge base to develop well detailed RAMI criteria is very sparse, especially regarding load conditions, material properties and component reliability. Simple deductions of design criteria from basic RAMI considerations turned out to be a valuable guideline for design improvements. Therefore, apart from the goal of fulfilling the system requirements, also RAMI considerations could be successfully used for design optimizations. Finally as the design approaches its final status, the importance of stable interface and technical requirement definitions is raising and becomes a key necessity for an efficient design progress.

\section{References}

[1] D. Strauss et al., Preliminary design of the ITER ECH Upper Launcher, In Press, Fusion Engineering and Design, Available online 11 May 2013

[2] Gandini, F. et al., The EC H\&CD transmission line for ITER, Fusion Science and Technology 59 (4) , pp. 709717, 2011

[3] T. Omori et al., Overview of the ITER EC H\&CD system and its capabilities, Fusion Engineering and Design 86 (6-8), 951-954, 2011

[4] Takahashi, K. et al., Progress of ITER equatorial electron cyclotron launcher design for physics optimization and toward final design, Fusion Engineering and Design 86 (6-8) , pp. 982-986, 2011

[5] Sauter, O. et al., Plasma Physics and Controlled Fusion 52 (2010) 025002

[6] T. A. Scherer et al., Experimental analysis of the inserted waveguide CVD diamond window prototype for the ITER ECRH upper launcher, 36th IRMMW-THz Conf., Houston, Texas (USA), IEEE-Proc., 2011

[7] G. Aiello et al., The ITER EC H\&CD Upper Launcher: Seismic Analysis, ISFNT 11, to be published, 2013

[8] Theo A. Scherer et al., Investigations of dielectric RF properties of ultra low loss CVD diamond disks for fusion applications, Proc. 34th Int. Conf. on IRMMWTHz Wave 2009, IEEE CFP09IMM-CDR, ISBN 978-14244-5417-4, Busan, Korea, 2009

[9] P. Spaeh, The ITER ECH \& CD Upper Launcher: Steps towards final design of the First Confinement System, Proc. SOFE 2013

[10] J. F. Koning, Analysis of ITER upper port plug remote handling maintenance scenarios, Fusion Engineering and Design 87(5-6), 2012

[11] D. Ronden, The ITER EC H\&CD Upper Launcher: Maintenance concepts, In Press, Fusion Engineering and Design, Available online 11 May 2013

[12] B. Weinhorst et al., Neutronic Analyses for the ITER electron cyclotron-heating upper launcher, ISFNT 11, to be published, 2013.

[13] A. Vaccaro et al., The ITER EC-H\&CD Upper Launcher: FEM Analyses of the Blanket Shield Module with respect to Surface and Nuclear Heat Loads, Proc. SOFE2013 Conference, San Francisco, 2013.

[14] A. Vaccaro et al., The ITER EC H\&CD upper launcher: EM disruption analyses, Fusion Engineering and Design, Available online 20 February 2013.

[15] D. Strauss et al, "Objectives and design of the launcher handling test facility at FZK," US-EU-JPN RF Heating Technology Workshop, Nara, J, September 26-30, 2006 